\title{
Antiferromagnetic interlayer exchange coupling across epitaxial, Ge-containing spacers
}

\author{
R. R. Gareev, ${ }^{\text {a) }}$ D. E. Bürgler, R. Schreiber, H. Braak, M. Buchmeier, and P. A. Grünberg \\ Institut für Festkörperforschung, Forschungszentrum Jülich GmbH, D-52425 Jülich, Germany
}

(Received 21 April 2003; accepted 1 July 2003)

\begin{abstract}
We give experimental evidence of antiferromagnetic interlayer exchange coupling of $\mathrm{Fe}(001)$ layers across epitaxial, Ge-containing spacers consisting of either Ge wedges embedded between two Si boundary layers or Si-Ge-multilayers. The coupling strengths are of the order of $1 \mathrm{~mJ} / \mathrm{m}^{2}$ and decay on a length scale below $2 \AA$ as determined from magneto-optic Kerr effect and Brillouin light scattering. The coupling evolves with the spacer thickness from ferromagnetic to prevailing $90^{\circ}$ or antiferromagnetic for $\mathrm{Ge}$ wedges and $\mathrm{Si}-\mathrm{Ge}$ multilayers, respectively. The bilinear coupling is comparable in both cases, but the biquadratic contribution is suppressed for $\mathrm{Si}-\mathrm{Ge}$-multilayer spacers. Thus, $\mathrm{Si}-\mathrm{Ge}-\mathrm{multilayer}$ spacers give rise to perfect antiparallel alignment of the Fe film magnetizations. (C) 2003 American Institute of Physics. [DOI: 10.1063/1.1606102]
\end{abstract}

Recent observations of antiferromagnetic interlayer exchange coupling (AFC) across nonconducting epitaxial spacers of nominally pure Si (Ref. 1) and of MgO (Ref. 2) focuses particular interest on this new class of highly resistive structures that exhibits nonoscillatory AFC. For nominally pure, highly resistive Si spacers with thickness near $8 \AA$ the total coupling strength exceeds $5 \mathrm{~mJ} / \mathrm{m}^{2}$ and thus turns out to be significantly larger than for metallic spacers. ${ }^{1}$ Additionally, current-perpendicular-to-plane (CPP) transport measurements of $\mathrm{Fe} / \mathrm{Si} / \mathrm{Fe}$ structures demonstrate that AFC coexists with transport via tunneling. ${ }^{3}$

The reasons to study Ge-containing spacers are (i) to investigate the existence and the properties of AFC across a semiconductor other than $\mathrm{Si}$ and (ii) an attempt to vary the tunneling transparency and AFC of an epitaxial, nonconducting barrier by using lattice-matched ferromagnetic (FM) electrodes and spacers containing a combination of $\mathrm{Si}$ and $\mathrm{Ge}$. As was reported previously ${ }^{4}$ epitaxial $\mathrm{Fe} / \mathrm{Ge} / \mathrm{Fe}$ structures reveal no evidence of AFC. Therefore, we use in this work AFC-mediating, epitaxial Si boundary layers (BLs) at the interfaces to Fe layers in order to avoid direct contact of $\mathrm{Ge}$ with $\mathrm{Fe}$ and thus the possible formation of magnetic $\mathrm{Fe}-\mathrm{Ge}$ compounds that prevent AFC. ${ }^{4}$ The lattice mismatch of about $4 \%$ between $\mathrm{Ge}\left(a_{\mathrm{Ge}}=5.66 \AA\right)$ and $\mathrm{Si}\left(a_{\mathrm{Si}}\right.$ $=5.43 \AA$ ) gives rise to Stransky-Krastanov (SK) growth mode: several monolayers of strained Ge grow epitaxially on $\mathrm{Si}$, and Ge hillocks form for larger Ge thickness. ${ }^{5}$ In order to avoid SK growth we try to stabilize the strain in the spacer by piling up thin layers of $\mathrm{Ge}$ and $\mathrm{Si}$.

We grow our $\mathrm{Fe} /$ spacer/Fe(001) structures in a molecular-beam epitaxy system using a $150 \mathrm{~nm}$ thick $\mathrm{Ag}(001)$ buffer system on $\mathrm{GaAs}(001) .{ }^{1}$ The layers forming the spacers are deposited at low deposition rates $(<0.1 \AA / \mathrm{s})$. Three types of spacers are prepared: (i) Ge wedges, (ii) Ge wedges embedded between two $4 \AA$ thick Si BLs at both the bottom and top interfaces to the Fe layers, and (iii) multilayers consisting of $N$ alternating $2 \AA$ thick Si and Ge sublayers.

${ }^{a)}$ Electronic mail: r.gareev@fz-juelich.de
The nominal thickness of the Ge wedges ranges from 0 to 15 $\AA$. All spacers including the boundary layers presented here and the top Fe layer are grown at room temperature (RT). Total thickness of the Si BL of $8 \AA$ is chosen to obtain highly resistive spacers, which reveal strong insulating-type AFC. ${ }^{1}$ CPP transport measurements of $\mathrm{Fe} / \mathrm{Si}(8 \AA) / \mathrm{Fe}$ structures using the technique described in Ref. 3 demonstrate the presence of a tunneling barrier with a height of about $0.1 \mathrm{eV}$, which is characteristic of a small-gap semiconductor. The in-plane crystalline structure of all layers is characterized by means of low-energy electron diffraction (LEED). Magnetic properties are measured by the magneto-optical Kerr effect (MOKE) in Voigt geometry and Brillouin light scattering (BLS). Bilinear and biquadratic coupling constants, $J_{1}$ and $J_{2}$, are determined by fitting the field dependence of MOKE and BLS data using the usual areal energy density expression,

$$
E_{\mathrm{ex}}=-J_{1} \cos (\theta)-J_{2} \cos ^{2}(\theta),
$$

to phenomenologically describe interlayer exchange coupling, where $\theta$ is the angle between the two Fe film magnetizations. The external magnetic field $(H<800 \mathrm{mT})$ is applied along an easy axis of $\mathrm{Fe}(001)$. Further details concerning preparation of the structures, their characterization, and the fitting procedures are described in Refs. 1, 3, and 6.

First, we prepare Fe/Ge-wedge/Fe trilayers without BLs or with only one BL at the bottom or top interface. In all these cases the MOKE hysteresis curves reveal 100\% remanent magnetization characteristic of zero or FM coupling. Most likely, strong diffusion at the interfaces leads to the formation of magnetic $\mathrm{Fe}-\mathrm{Ge}$ compounds in the spacer, which cannot mediate AFC. ${ }^{4}$

In order to prevent $\mathrm{Fe}-\mathrm{Ge}$ interdiffusion we deposit a $\mathrm{Si}$ BL at both interfaces. Figure 1 shows typical LEED patterns for all five layers. The bottom Si BL grows at RT with a similar in-plane lattice constant as $\mathrm{Fe}(001)$. The nominally pure Ge spacers start to grow epitaxially on $\mathrm{Si}$ with an inplane cubic structure and a lattice parameter of about $2.9 \AA$ $\left(a_{\mathrm{Fe}}=2.87 \AA\right)$. For Ge thicknesses $t<6 \AA$ we observe 


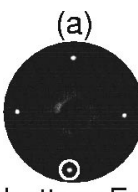

bottom $\mathrm{Fe}$
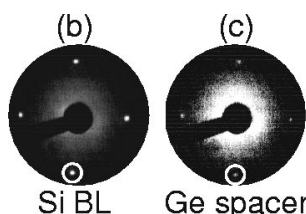

Ge spacer
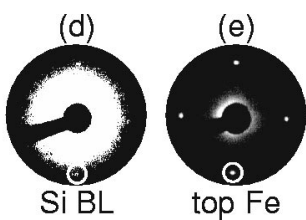

FIG. 1. LEED patterns taken at $55 \mathrm{eV}$ for a (a) $50 \AA$ bottom Fe layer, (b) 4 $\AA \mathrm{Si}$ bottom BL, (c) $5 \AA$ Ge spacer, (d) $4 \AA$ Si top BL, and (e) $30 \AA$ top Fe layer. The Si BL, Ge spacer, and top Fe layer are grown at RT. Circles mark the positions of the (01) spot of body-centered-cubic $\mathrm{Fe}(001)$ corresponding to an in-plane lattice constant of $2.9 \AA$.

LEED patterns for the Ge spacer, the top BL, and the top Fe layer. Thus, we obtain epitaxial growth of the whole structure.

In Fig. 2 we present the typical thickness dependence of the remanent magnetization normalized to the saturation magnetization $M_{r} / M_{s}$ and the saturation field $H_{s}$ for structures with Ge wedges embedded between two Si BLs. Four regions with different interlayer coupling behavior can be distinguished: (1) strong AFC for $t<4 \AA$, (2) increasing $90^{\circ}$ coupling for $4 \AA<t<6 \AA$, (3) almost pure $90^{\circ}$ coupling for 6 $\AA<t<10 \AA$ and, finally (4) negligible AFC or FM coupling for $t>10 \AA$.

Figure 3 shows the dependence of the interlayer exchange coupling constants $J_{1}$ and $J_{2}$ on the thickness of the Ge spacer $t$, again for a sample with two BLs. The values of $J_{1}$ and $J_{2}$ are obtained by fitting the experimental field dependence of BLS acoustic and optical spin-wave modes as described in Ref. 6. The bilinear coupling constant $J_{1}$ quickly drops with $t$ and can be described by exponential decay with a decay length of about $t_{0}=1.5 \AA$ (dashed line in Fig. 3). The biquadratic term $\left|J_{2}\right|$ is smaller than $\left|J_{1}\right|$ only for $t<3 \AA$. However, the biquadratic coupling is dominant for all Ge thicknesses because $\left|J_{1}\right|<2\left|J_{2}\right|$ (see the inset of Fig. 3), and the layer magnetizations are canted in the remanent state, even for the smallest $t$. The data in Fig. 3 confirm the qualitative interpretation of Fig. 2 given above. The decay of $J_{1}$ is very similar to that in the case of nominally pure $\mathrm{Si}$ wedges $\left(t_{0}=1.7 \AA\right.$ ), whereas the $J_{2}$ behavior is opposite that of the Si case: ${ }^{1} J_{1} / J_{2}$ decreases for Ge wedges, but it increases for $\mathrm{Si}$ wedges. The similar behavior of the bilinear coupling indicates its intrinsic origin. The likely rea-

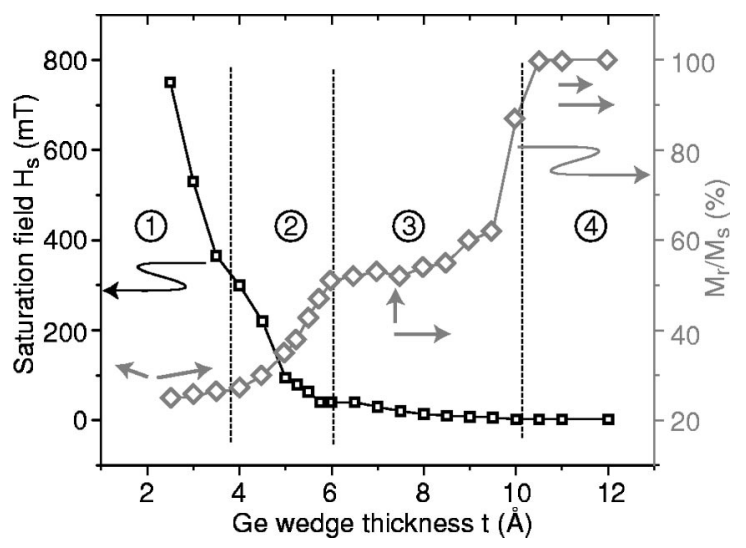

FIG. 2. $M_{r} / M_{s}$ and $H_{s}$ vs nominal Ge thickness $t$ for a wedge-type Fe (50 $\AA) / \mathrm{Si} / \mathrm{Ge}(t) / \mathrm{Si} / \mathrm{Fe}(30 \AA)$ structure with a $4 \AA$ thick $\mathrm{Si} \mathrm{BL}$ derived from MOKE hysteresis loops. The arrows indicate the relative magnetization

wedge-type $\mathrm{Fe}(50 \AA) / \mathrm{Si} / \mathrm{Ge}(t) / \mathrm{Si} / \mathrm{Fe}(30 \AA)$ sample at $t=2.5 \AA$.
alignment in the remanent state.
Downloaded 21 Dec 2006 to 134.94 .122 .39 . Redistribution subject to AIP license or copyright, see http://apl.aip.org/apl/copyrigh

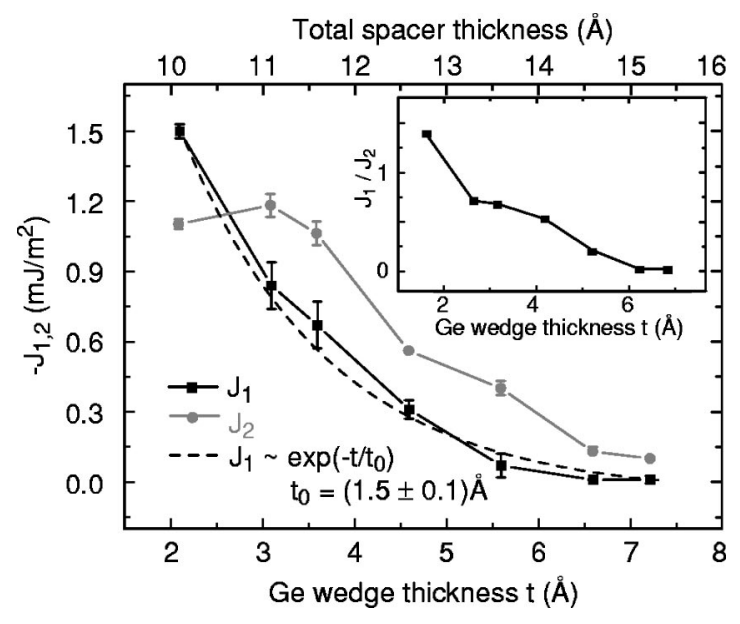

FIG. 3. Bilinear $\left(J_{1}\right)$ (black) and biquadratic $\left(J_{2}\right)$ (gray) coupling constants vs nominal Ge thickness $t$ for a wedge-type $\mathrm{Fe}(50 \AA) / \mathrm{Si} / \mathrm{Ge}(t) / \mathrm{Si} / \mathrm{Fe}(30 \AA)$ structure with $4 \AA$ thick Si BLs. The dashed line is an exponential fit and yields a decay length of about $1.5 \AA$. The upper abscissa gives the total spacer thickness including the Si BLs. The inset shows the ratio of $J_{1} / J_{2}$ vs $t$.

son for the observed strong biquadratic coupling is the competition between bilinear coupling and FM coupling due to magnetic bridges. ${ }^{7}$ The onset of SK hillock growth at Ge thicknesses $t>6 \AA$ reduces the efficiency of FM bridge annihilation upon further Ge deposition. The thickness dependence of $J_{2}$ is in this scenario related to degradation of the structural quality of Ge with thickness $t$. In this context, it is interesting to note that the vanishing of $J_{1}$ and the prevalence of $J_{2}$ coincide with the disappearance of the LEED spots. As was the case for the $\mathrm{Fe} / \mathrm{Si} / \mathrm{Fe}$ structures, ${ }^{1}$ the existence of strong AFC is closely related to good crystalline order in the spacer.

Therefore, we prepare in the next step samples of the form $\mathrm{Fe} / \mathrm{Si} / \mathrm{Ge}, \ldots$, , $\mathrm{Fe}$ with $N$ alternating $\mathrm{Si}$ and $\mathrm{Ge}$ sublayers (Si-Ge multilayers) as spacers. We observe LEED patterns similar in quality to those in Fig. 1, even for larger spacer thicknesses than the Ge wedge. Figure 4 depicts as an example the MOKE hysteresis loop of a sample with $N=6$. The gray line is a fit that yields $J_{1}$ and $J_{2}$ as indicated. $J_{1}$ is dominant $\left(\left|J_{1}\right|>2\left|J_{2}\right|\right)$, and thus the hysteresis loop exhibits a plateau for $|H|<30 \mathrm{mT}$ due to perfect antiparallel align-

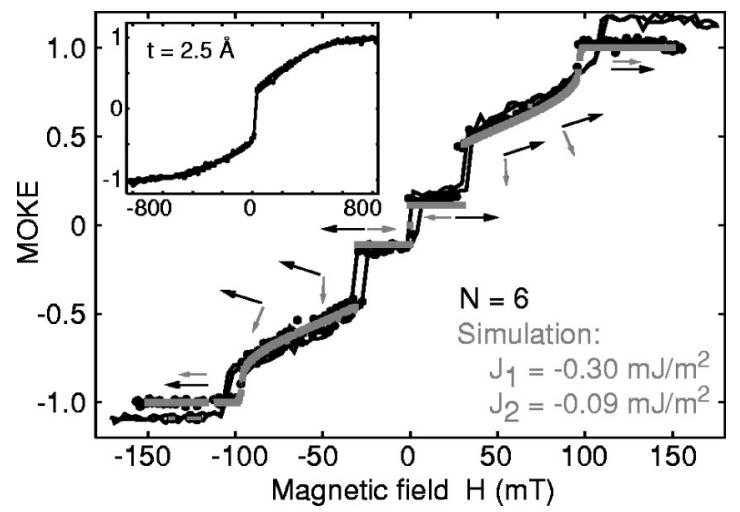

FIG. 4. Experimental (black) and fitted (gray) MOKE hysteresis loops of a

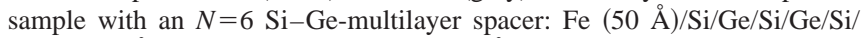
$\mathrm{Ge} / \mathrm{Fe}(30 \AA)$. All Si and Ge layers are $2 \AA$ thick. The arrows indicate the magnetization alignment obtained from fitting. Inset: Hysteresis loop of a wedge-type $\mathrm{Fe}(50 \AA) / \mathrm{Si} / \mathrm{Ge}(t) / \mathrm{Si} / \mathrm{Fe}(30 \AA)$ sample at $t=2.5 \AA$. 


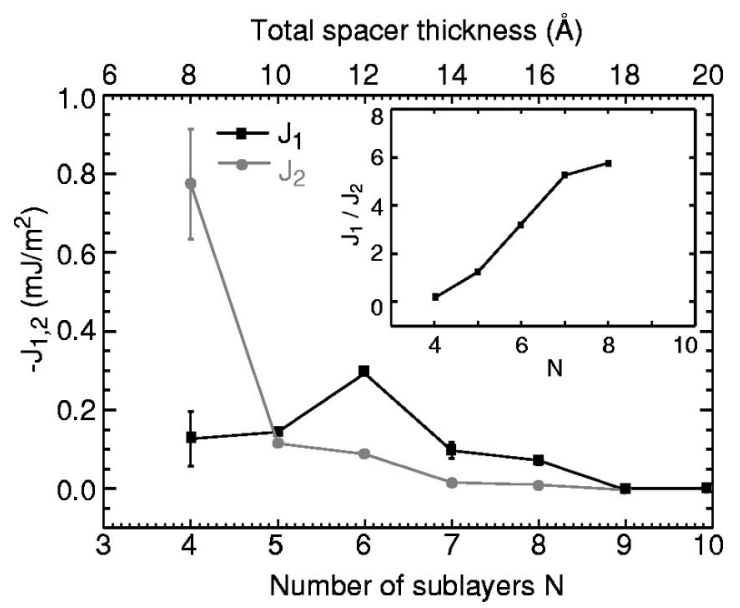

FIG. 5. Bilinear $\left(J_{1}\right)$ (black) and biquadratic $\left(J_{2}\right)$ (gray) coupling constants vs the number $N$ of $2 \AA$ thick $\mathrm{Si}$ and Ge sublayers in the multilayer spacer for structures of $\mathrm{Fe}(50 \AA) / \mathrm{Si} / \mathrm{Ge}, \ldots, / \mathrm{Fe}(30 \AA)$. The upper abscissa gives the total spacer thickness in $\AA$. The inset shows the ratio of $J_{1} / J_{2}$ vs $t$.

ment (shown by arrows in Fig. 4). The step at $H=0$ arises from the different Fe thicknesses. Such a plateau is not observed for the samples with Ge embedded between BLs (inset of Fig. 4). In Fig. 5 we present the dependence of the coupling constants derived from fitting MOKE loops on the number $N$ of sublayers. The interlayer coupling is FM for smallest spacer thicknesses $(N<4)$ and becomes due to FM bridges prevalent $90^{\circ}$ coupling for $N=4$, where $J_{2} \approx 6 J_{1}$. For $N=5, J_{1}$ and $J_{2}$ are comparable. The increase of $J_{1}$ from $N=4$ to 6 and the simultaneous steep decrease of $J_{2}$ are due to the transition from FM coupling first to competing FM and antiferromagnetic interactions, giving rise to $90^{\circ}$ coupling $^{7}$ and further to dominant AFC for $N=6-8$. Finally, for $N=9 \mathrm{AFC}$ is negligible, and both FM layers are aligned parallel to field $H$.

The maximum of $\left|J_{1}\right|$ of about $0.3 \mathrm{~mJ} / \mathrm{m}^{2}$ is reached for $N=6$, corresponding to a total spacer thickness of $12 \AA$. For the Ge-wedge samples taking the thickness of the BL into account, a total spacer thickness of $12 \AA$ corresponds to $t=4$ $\AA$. Thus, the strength of the bilinear coupling across the $\mathrm{Si}-$ Ge-multilayer is comparable to that across embedded $\mathrm{Ge}$ wedges of similar total thickness (Fig. 3). The bilinear coupling extends to about the same total spacer thicknesses: 16 $\AA(N=8)$ for the multilayer spacers and $15 \AA(t=7 \AA)$ for the embedded Ge wedges (Fig. 3). However, the evolution of $J_{2}$ is completely different for the two types of samples: for multilayer spacers it is very strong for small $N$ due to strong intermixing, but drops rapidly and becomes secondary for larger $N$. This different behavior is best recognized by comparing the dependence of the $J_{1} / J_{2}$ ratios in the insets of Figs. 3 and 5. We relate these observations to the wellestablished mechanism of strain stabilization in $\mathrm{Si} / \mathrm{Ge}$ multilayers $^{8}$ that shifts the transition to SK hillock growth to larger thicknesses, as indicated by our LEED data. Thus, the multilayer spacers show improved growth compared to the Ge-wedge samples, once they are thick enough $(N>5)$ to sufficiently suppress the formation of FM bridges.

We have measured the vertical stray fields over the samples generated from FM bridges using a superconducting quantum interference device (SQUID) microscope. ${ }^{9,10}$ The images show features at all spacer thicknesses where we observe domainant $J_{2}$ and are structureless where $J_{1}$ prevails, ${ }^{10}$ consistent with the above interpretation.

In conclusion, we have shown that epitaxial $\mathrm{Fe} / \mathrm{spacer} / \mathrm{Fe}$ structures containing Ge in the spacer demonstrate AFC by studying nominally pure Ge wedges embedded between two $\mathrm{Si}$ BLs and $\mathrm{Si}-\mathrm{Ge}-\mathrm{multilayer}$ spacers. The coupling strengths are of the order of $1 \mathrm{~mJ} / \mathrm{m}^{2}$ and decay on a length scale of less than $2 \AA$. Biquadratic coupling is observed for both sample types at small spacer thicknesses. However, the biquadratic contribution vanishes much faster for the multilayer spacers as evidenced by the different behavior of the $J_{1} / J_{2}$ ratios. We believe that this is due to strain stabilization ${ }^{8}$ in the layered structure. The resulting better growth quality for sufficiently large $N$ with a suppressed impact of FM bridges on the coupling gives rise to almost pure bilinear coupling with perfect antiparallel alignment. The concept of inserting AFC-mediating epitaxial BLs at interfaces to reduce interdiffusion has proven successful because no evidence of AFC or biquadratic coupling could be observed for structures without BLs. These results indicate that relatively strong AFC is a common feature of wellordered, epitaxial spacer layers that consist of semiconducting elements. A quantitative theoretical description of strong AFC across semiconductor spacers-representing the intermediate case between metallic and insulating spacers-is highly desired.

This work was supported by the Strategiefondsprojekt "Magnetoelectronics" of the Helmholtz-Gemeinschaft Deutscher Forschungszentren (HGF).

${ }^{1}$ R. R. Gareev, D. E. Bürgler, M. Buchmeier, R. Schreiber, and P. Grünberg, J. Magn. Magn. Mater. 240, 235 (2002).

${ }^{2}$ J. Faure-Vincent, C. Tiusan, C. Bellouard, E. Popova, M. Hehn, F. Montaigne, and A. Schuhl, Phys. Rev. Lett. 89, 107206 (2002).

${ }^{3}$ R. R. Gareev, L. L. Pohlmann, S. Stein, D. E. Bürgler, P. A. Grünberg, and M. Siegel, J. Appl. Phys. 93, 8038 (2003).

${ }^{4}$ J. J. de Vries, J. Kohlhepp, F. J. A. den Broeder, P. A. Verhaegh, R. Jungblut, A. Reinders, and W. J. M. de Jonge, J. Magn. Magn. Mater. 165, 435 (1997).

${ }^{5}$ Y.-W. Mo, D. E. Savage, B. S. Swartzentruber, and M. G. Lagally, Phys. Rev. Lett. 65, 1020 (1990).

${ }^{6}$ M. Buchmeier, B. K. Kuanr, R. R. Gareev, D. E. Bürgler, and P. Grünberg, Phys. Rev. B 67, 184404 (2003).

${ }^{7}$ J. C. Slonczewski, J. Appl. Phys. 75, 6474 (1994).

${ }^{8}$ S. C. Jain and W. Hayes, Semicond. Sci. Technol. 6, 547 (1991).

${ }^{9}$ J. R. Kirtley, Physica C 368, 55 (2002).

${ }^{10} \mathrm{U}$. Poppe (unpublished) 